\title{
Adsorption characteristics of gallic acid on fresh calcium phosphate in sucrose solution
}

\author{
TIAN Yu-hong ${ }^{1, \text { a }, ~ X U ~ X i a n g-l i ~}{ }^{1}$, WU Shang-guang ${ }^{1}$, WEI Qi-qiu ${ }^{1}$ and CHENG \\ $\mathrm{HaO}^{1}$
}

\author{
${ }^{1}$ College of Biological and Chemical Engineering, Guangxi University of Science and Technology, \\ Liuzhou 545006, China \\ atianyuhonglz@163.com
}

\begin{abstract}
Keywords: fresh calcium phosphate; gallic acid; adsorption kinetics; adsorption isotherm.
Abstract. The adsorption characteristics of gallic acid on fresh calcium phosphate in sucrose solution were discussed. Three kinetic models, including pseudo-first-order model, pseudo-second-order model and intraparticle diffusion model, were selected to follow the adsorption processes. The results which adsorption reaction occurred in $10 \%$ sucrose solution showed that the pseudo-second-order equation provided the best correlation to the experimental data $\left(R^{2}=0.9908 \sim 0.9964\right)$. Under the initial gallic acid concentration were $25,50,75 \mathrm{mg} \cdot \mathrm{L}^{-1}$ in the $10.0 \%$ sucrose solution, the adsorption rate of gallic acid on fresh calcium phosphate were $0.0614,0.0368,0.0149 \mathrm{~g} \cdot \mathrm{mg}^{-1} \cdot \mathrm{min}^{-1}$, respectively. Adsorption isotherms curves of gallic acid from different concentrations of sucrose solution on fresh calcium phosphate all conforms to the Langmuir model $\left(\mathrm{R}^{2}=0.9919 \sim 0.9965\right)$. The sucrose solution concentration had effect on the saturated adsorption capacity of gallic acid. The maximum adsorption capacity of gallic acid were $8.4317,8.0906,7.7160,7.3964,7.0872,7.0771 \mathrm{mg} \cdot \mathrm{g}^{-1}$ as sucrose concentrations were $0.0 \%, 0.1 \%, 0.5 \%, 1.0 \%, 5.0 \%$ and $10.0 \%$, respectively.
\end{abstract}

\section{Introduction}

In the process of cane sugar manufacturing, how to remove the colored substances contained in the products and inhibit the generation of new colored substances is one of the key factors to ensure the quality of finished sugar. The original color of sugar-cane juice is not deep, however, the color value of the mixed juice after extraction is very high and the main reason is the color increasing reaction in production process: catalytic oxidation of phenolic substances into dark substance by phenolase, the iron in equipment can dissolve in sugar-cane juice under the aerobic and acidic condition and then react with phenolic substances and then generate dark iron complex, the reducing sugars and amino acids in sugarcane juice have a reaction and produce Maillard Reactant. Therefore, the effect of removing phenolic substances is the important factor of deciding the sucrose crystal color.

Sulphurous acid method clarification technique, because of the characteristics of less equipment, simple technological process and convenient management, gets a more extensive application in sugar industry in China. For the sulphurous acid method clarification technique, the adsorption and flocculation function of calcium phosphate and calcium sulfite plays an important role in the decoloration of sugarcane juice. Calcium phosphate is one of the most commonly used solid detergent, due to its excellent properties, such as, non-toxic, high chemical and thermal stability and high biocompatibility etc. have become the research hotspot [1-3]. Gallic acid is one of the phenolic substances with a large number of content in the sugar-cane, presently, the research on the adsorption of gallic acid by calcium phosphate has not been reported. In this paper, it has taken experimental study of the adsorption performance of new calcium phosphate for gallic acid and it has strengthened the basic research of sugarcane juice and molasses decoloration.

\section{Materials and methods}

Materials and equipment. Gallic acid standard was purchased from National Institutes for Food and Drug Control (Beijing, China ). Gallic acid(AR) sample was provided from Tianjin Guangfu Fine 
Chemical Research Institute (Tianjin, China). Calcium hydroxide(AR) and sodium dihydrogen phosphate(AR) were obtained from Xilong Chemical Co., Ltd (Shantou, China ). phosphoric acid(AR), sodium hydroxide(AR), hydrochloric acid(AR) and sodium carbonate(AR) were purchased from Guangdong Guanghua Chemical Factory Co., Ltd (Shantou, China ). Folin-phenol reagent was purchased from Sinopharm Chemical Reagent Co., Ltd (Shanghai, China ). Double distilled water.

Electronic analytical balance ( AB104-N, Mettler-Toledo instrument Co., Ltd; Shanghai, China). pH meter (S-25, Shanghai Lida Instrument Factory; Shanghai, China). Digital constant temperature water bath pot (HH-6, Guohua Electric Appliance Co., Ltd; Changzhou, China). Constant temperature vibrator (SHZ-82A, Guohua Electric Appliance Co., Ltd; Changzhou, China). Automatic triple water distiller (SZ-97, Shanghai Yarong Biochemical Instrument Plant; Shanghai, China). Visible spectrophotometer (722, Shanghai Precision and Scientific Instrument Co., Ltd; Shanghai, China).

Analysis and Detection Method for Gallic Acid in Sugar Solution System. Accurately take $0.0050 \mathrm{~g}$ gallic acid and add distilled water to dissolve and then transfer it into $100 \mathrm{~mL}$ volumetric flask for constant volume, and then get $50 \mathrm{mg} \cdot \mathrm{L}^{-1}$ of gallic acid standard solution, lately respectively take $0.00,2.00,4.00,6.00,8.00,10.00 \mathrm{~mL}$ of $50 \mathrm{mg} \cdot \mathrm{L}^{-1}$ of gallic acid solution into $50 \mathrm{~mL}$ volumetric flask, and then add respectively $1.50 \mathrm{~mL}$ forint phenol reagent and shake well, after $3 \sim 8$ min's standing, add $5 \mathrm{~mL} 7.5 \%$ sodium carbonate solution and use distilled water to dilute it to the scale and then shake well to get the color $1 \mathrm{~h}$. Measure the absorbance value at the place of $765 \mathrm{~nm}$, with absorbance value as the ordinate and with the gallic acid content in standard solution as abscissa, and then make out the linear regression curve to measure the gallic acid content in filtrate[4]. With 10\% sucrose solution as the blank.

Preparation of fresh calcium phosphate. Accurately weigh and take $10.00 \mathrm{~g} \mathrm{Ca}(\mathrm{OH})_{2}$ into 500 $\mathrm{mL}$ beaker and add $200 \mathrm{~mL}$ distilled water to make lime milk, stirring and dropwise adding $10 \%$ $\mathrm{H}_{3} \mathrm{PO}_{4}$ so that to get the $\mathrm{pH}=7.00 \pm 0.01$. Keep stirring for $30 \mathrm{~min}$ and then to take the suction filtration.

Adsorption kinetic. Accurately weigh and take $1.50 \mathrm{~g}$ fresh calcium phosphate samples into a series of $250 \mathrm{~mL}$ conical flask with cover and then add the configured $100 \mathrm{~mL}$ gallic acid sugarcane solution with a certain concentration (gallic acid concentration of $25,50,75 \mathrm{mg} \cdot \mathrm{L}^{-1}$ respectively, sucrose concentration of $10 \%$ ), lately use $\mathrm{HCl}$ and $\mathrm{NaOH}$ solution to preliminary adjust the $\mathrm{pH}$ of the system. Vibrate the reaction system in the temperature of $30^{\circ} \mathrm{C}$ with the oscillation frequency of 120 $\mathrm{r} / \mathrm{min}$ for $5,10,15,30,45,60,80,100,150,200,300,400 \mathrm{~min}$, and after filtrating with $0.22 \mu \mathrm{m}$ filter membrane, taking $10 \%$ sucrose solution as the blank and then rapidly measure the concentration and $\mathrm{pH}$ of gallic acid in the filtrate and calculate the adsorption quantity with the no added fresh calcium phosphate system as reference .

The adsorption capacity was calculated according to the following equation:

$$
q=\frac{V\left(C_{0}-C\right)}{1000 m}
$$

where $q$ is adsorption capacity of gallic acid on fresh calcium phosphate $\left(\mathrm{mg} \cdot \mathrm{g}^{-1}\right) ; V$ is the volume of gallic acid solution $(\mathrm{mL}) . C_{0}$ is the concentration of gallic acid in control group $\left(\mathrm{mg} \cdot \mathrm{L}^{-1}\right), C_{\mathrm{e}}$ is the equilibrium gallic acid concentration in solution $\left(\mathrm{mg} \cdot \mathrm{g}^{-1}\right), \mathrm{m}$ is the mass of fresh calcium phosphate used (g).

Adsorption kinetics model The dynamic behavior of the adsorption was examined by fitting the dynamic data with three kinetic models, including intraparticle diffusion models, pseudo-first order model and pseudo-second order model.

The linearized form of the intraparticle diffusion rate equation is given by Weber-Morris as[5-6]:

$$
q_{t}=k_{p} t^{0.5}
$$

where $q_{\mathrm{t}}$ is the adsorption capacity at time $t\left(\mathrm{mg} \cdot \mathrm{g}^{-1}\right) \cdot k_{p}$ is the intraparticle diffusion rate constant $\left(\mathrm{mg} \cdot \mathrm{g}^{-1} \cdot \mathrm{min}^{-0.5}\right)$, The values $k_{p}$ correlation coefficient calculated from the slope of the plots of $q_{\mathrm{t}}$ versus $t^{0.5}$ 
The kinetic data were further analyzed using the pseudo-first order kinetics model. A linear form is given as[5-7]:

$$
\ln \left(q_{e}-q_{t}\right)=\ln q_{e}-k_{1} t
$$

where $q_{\mathrm{e}}$ is the equilibrium adsorption capacity . $k_{1}$ is the rate constant of the pseudo-first adsorption $\left(\mathrm{min}^{-1}\right)$.

The kinetic data can also be analyzed by the pseudo-second order kinetics model, formulated as[5-7]:

$$
\frac{t}{q_{t}}=\frac{1}{k_{2} q_{e}^{2}}+\frac{t}{q_{e}}
$$

where $k_{2}$ is the pseudo-second order rate constant $\left(\mathrm{g} \cdot \mathrm{mg}^{-1} \cdot \mathrm{min}^{-1}\right)$.

Adsorption isotherms. Configure systematical concentration's gallic acid solution separately in the $0.0 \%, 0.1 \%, 0.5 \%, 1.0 \%, 5.0 \%$ and $10.0 \%$ of sucrose solution to make the concentration of gallic acid systematically and evenly distribute in $25 \sim 1000 \mathrm{mg} \cdot \mathrm{L}^{-1}$. Separately add the configured $100 \mathrm{~mL}$ gallic acid sugarcane solution with systematical concentration into $250 \mathrm{~mL}$ conical flask with cover and accurately weigh and take $1.50 \mathrm{~g}$ fresh calcium phosphate samples into conical flask, lately use $\mathrm{NaOH}$ and $\mathrm{HCl}$ solution to preliminarily regulate system $\mathrm{pH}$. Vibrate the reaction system in the temperature of $30^{\circ} \mathrm{C}$ with the oscillation frequency of $120 \mathrm{r} / \mathrm{min}$ for $150 \mathrm{~min}$, and after filtrating with $0.22 \mu \mathrm{m}$ filter membrane to rapidly measure the concentration and $\mathrm{pH}$ of gallic acid in the filtrate and calculate the adsorption quantity with the no added new calcium phosphate system as reference .

Adsorption isotherm model. In order to examine relationship between the equilibrium concentration of gallic acid on fresh calcium phosphate and the equilibrium adsorption capacity, as well as to understand the maximum adsorbing capacity of the adsorption systems. Hence, the Freundlich and Langmuir equations were used to fit the equilibrium isotherms.

The linear form of the Langmuir isotherm equation is written as[8-9]:

$$
\frac{C_{e}}{q_{e}}=\frac{C_{e}}{b}+\frac{1}{k b}
$$

where $C_{e}$ is the equilibrium concentration of solution $\left(\mathrm{mg} \cdot \mathrm{L}^{-1}\right), b$ is the monolayer adsorption capacity of the adsorbent $\left(\mathrm{mg} \cdot \mathrm{g}^{-1}\right)$, and $k$ is the Langmuir adsorption constant.

The linear form of the Freundlich isotherm equation is written as[9-10]:

$\ln q_{e}=\ln k+n \ln C_{e}$

where $k$ and $n$ are the Freundlich adsorption isotherm constants.

\section{Result and Discussion}

Draw of Standard Curve. According to the law of Lambert-beer, taking the absorbancy(Y) of gallic acid as the ordinate and the quality concentration $(\mathrm{X})$ of gallic acid as the abscissa to draw the standard curve. The calculated regression equation: $\mathrm{Y}=0.0925+0.023 \mathrm{X}$, correlation coefficient $\mathrm{R}^{2}$ $=0.9979$, the quality concentration of gallic acid presents good linear relationship in the range of $0 \sim$ $10 \mathrm{mg} \cdot \mathrm{L}^{-1}$

Adsorption kinetic of gallic acid on fresh calcium phosphate. Under the situation that temperature of $30^{\circ} \mathrm{C}, \mathrm{pH}=7.3 \pm 0.1$, sucrose concentration of $10 \%$, gallic acid concentration of 25,50 , $75 \mathrm{mg} \cdot \mathrm{L}^{-1}$, separately, the adsorption kinetics curve of fresh calcium phosphate for gallic acid is shown in figure 1.

From figure 1, we can know that, at the beginning of adsorption, adsorption quantity of fresh calcium phosphate for gallic acid increases with the extension of adsorption time, when the adsorption time reaches to $150 \mathrm{~min}$, the adsorption of fresh calcium phosphate for gallic acid is in a quilibrium state and followed the adsorption quantity will no longer increase with the extension of reaction time. In the aqueous solution without sucrose, the adsorption speed of fresh calcium phosphate for gallic acid is higher than in sucrose solution and the adsorption equilibrium time is 100 $\min [11]$. According to the experimental methods, separately adopt the intraparticle diffusion model, 
pseudo-first order kinetic model and pseudo-second order kinetic model to take fitting for the adsorption kinetics curve data of fresh calcium phosphate for gallic acid and the fitting results is shown in figure $2 \sim 4$ and table 1.

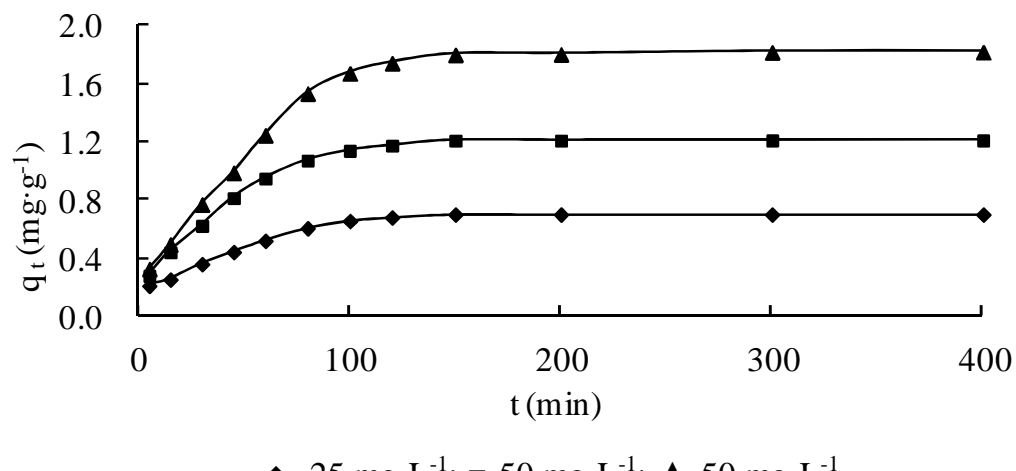

- $-25 \mathrm{mg} \cdot \mathrm{L}^{-1} ; \mathbf{-}-50 \mathrm{mg} \cdot \mathrm{L}^{-1} ; \boldsymbol{\Delta}-50 \mathrm{mg} \cdot \mathrm{L}^{-1}$

Fig.1 Adsorption kinetic curves of gallic acid on fresh calcium phosphate with $10 \%$ sucrose

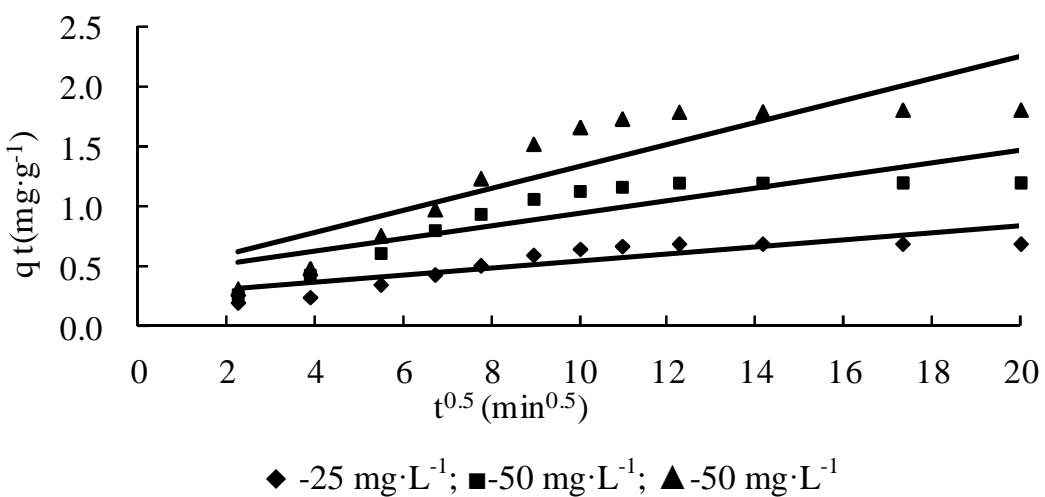

Fig.2 Intraparticle diffusion equation of gallic acid on fresh calcium phosphate with $10 \%$ sucrose

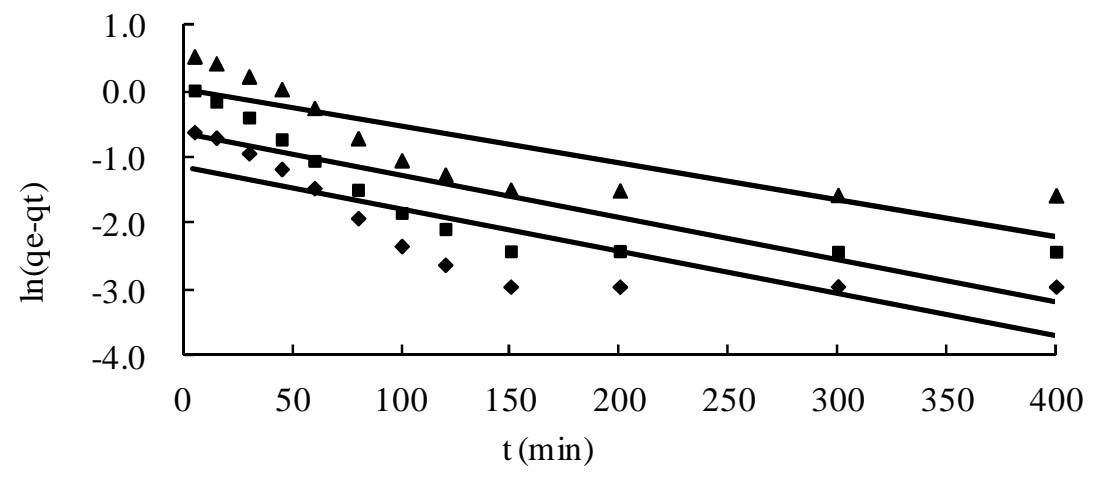

$-25 \mathrm{mg} \cdot \mathrm{L}^{-1} ; \mathbf{-}-50 \mathrm{mg} \cdot \mathrm{L}^{-1} ; \mathbf{\Lambda}-50 \mathrm{mg} \cdot \mathrm{L}^{-1}$

Fig.3 Pseudo-first-order reaction equation of gallic acid on fresh calcium phosphate with $10 \%$ sucrose

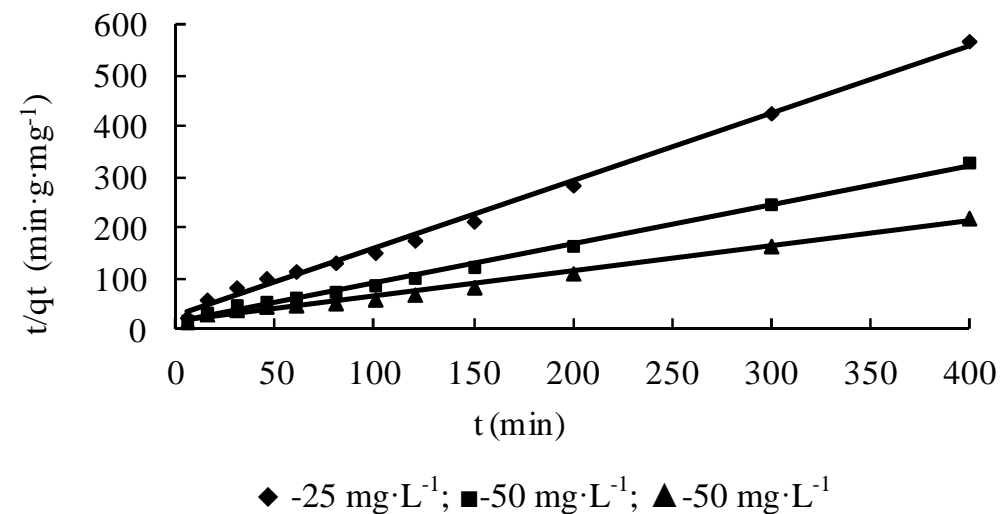

Fig.4 Pseudo-second-order reaction equation of gallic acid on fresh calcium phosphate with $10 \%$ sucrose 
Table 1 Adsorption kinetics constant of gallic acid on fresh calcium phosphate with $10 \%$ sucrose

\begin{tabular}{|c|c|c|c|c|c|c|c|}
\hline \multirow{2}{*}{$\begin{array}{l}\text { Initial gallic } \\
\text { acid } \\
\text { concentrtion } \\
\left(\mathrm{mg} \cdot \mathrm{L}^{-1}\right)\end{array}$} & \multicolumn{2}{|c|}{ intraparticle diffusion model } & \multicolumn{2}{|c|}{$\begin{array}{c}\text { pseudo-first-order } \\
\text { model }\end{array}$} & \multicolumn{3}{|c|}{ pseudo-second-order model } \\
\hline & $\begin{array}{c}\mathrm{K}_{\mathrm{p}} \\
\left(\mathrm{mg} \cdot \mathrm{g}^{-1} \cdot \min ^{-0.5}\right)\end{array}$ & $\mathrm{R}^{2}$ & $\begin{array}{c}\mathrm{K}_{1} \\
\left(\min ^{-1}\right)\end{array}$ & $\mathrm{R}^{2}$ & $\begin{array}{c}\mathrm{q}_{\mathrm{e}} \\
\left(\mathrm{mg} \cdot \mathrm{g}^{-1}\right)\end{array}$ & $\begin{array}{c}\mathrm{K}_{2} \\
\left(\mathrm{~g} \cdot \mathrm{mg}^{-1} \cdot \min ^{-1}\right)\end{array}$ & $\mathrm{R}^{2}$ \\
\hline 25 & 0.0301 & 0.7535 & 0.0064 & 0.6653 & 0.7556 & 0.0614 & 0.9955 \\
\hline 50 & 0.0530 & 0.7249 & 0.0063 & 0.6530 & 1.3024 & 0.0368 & 0.9964 \\
\hline 75 & 0.0910 & 0.7625 & 0.0056 & 0.6728 & 2.0288 & 0.0149 & 0.9908 \\
\hline
\end{tabular}

From the fitting parameters in figure 2, 3, 4 and table 1, you can see that the pseudo-second-order equation provided the best correlation to the experimental data $\left(R^{2}=0.9908 \sim 0.9964\right)$. Through the fitted equation of pseudo-second order kinetics to calculate out when the initial concentration of gallic acid is $25,50,75 \mathrm{mg} \cdot \mathrm{L}^{-1}$ respectively in experimental conditions, the adsorption rate constant of fresh calcium phosphate for gallic acid in $10 \%$ sucrose solution is $0.0614,0.0368,0.0149 \mathrm{~g} \cdot \mathrm{mg}^{-1} \cdot \mathrm{min}^{-1}$ and its theoretical adsorption quantity separately are $0.7556,1.3024$ and $2.0288 \mathrm{mg} \cdot \mathrm{g}^{-1}$.

In the solution without sucrose, the adsorption of fresh calcium phosphate for gallic acid also conforms to the pseudo-second order kinetic equation, the range of correlation coefficient $\mathrm{R}^{2}$ is $0.9943 \sim 0.9946$, which shows that the existing of sugar molecules does not influence the adsorption dynamics model of fresh calcium phosphate[11]. Compared with the adsorption experiment without sucrose solution, the adsorption rate of fresh calcium phosphate for gallic acid will decrease due to the existing of sucrose, which is manifested on that the time of reaching to the adsorption equilibrium state for the adsorption of fresh calcium phosphate for gallic acid in sucrose solution system has certain extension. The main reason is that sucrose increases the viscosity of solution and slows the movement speed of gallic acid, as a result that the time for gallic acid to reach to the surface of fresh calcium phosphate is extended, thus the adsorption rate is reduced.

Adsorption Isotherm. In the experimental conditions of temperature of $30^{\circ} \mathrm{C}$, adsorption time of $150 \mathrm{~min}$ and $\mathrm{pH}$ of $7.30 \pm 0.1$, the adsorption isotherm of gallic acid by fresh calcium phosphate in different concentration of sucrose solution system is shown in figure 5.

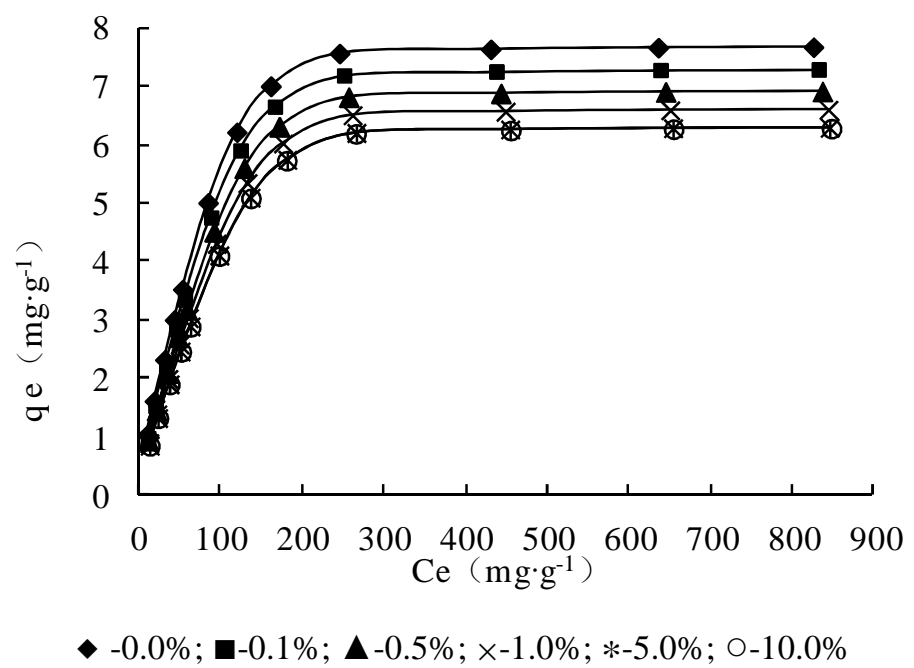

Fig.5 Adsorption curve of gallic acid on fresh calcium phosphate in different concentrations of sucrose

The figure 5 shows that in the range of low quality of gallic acid concentration, the adsorption quantity of fresh calcium phosphate for gallic acid increases quickly with the rising of equilibrium concentration, when the equilibrium concentration of gallic acid is bigger than $200 \mathrm{mg} \cdot \mathrm{L}^{-1}$, the increase of adsorption quantity becomes slow. When the equilibrium concentration of gallic acid concentration reaches to $300 \mathrm{mg} \cdot \mathrm{L}^{-1}$, the adsorption quantity of fresh calcium phosphate for gallic acid is saturated and if continue to increase the concentration of gallic acid solution, the adsorption quantity will no longer increase.

It can be seen from the figure 5 that, sucrose concentration has certain influence on the adsorption quantity of fresh calcium phosphate for gallic acid, besides, the adsorption quantity of that in the 
system containing no sugar is the largest. With the increase of sucrose solution concentration, the adsorption quantity of fresh calcium phosphate for gallic acid gradually reduces. Separately use Langmuir isothermal equation and the Freundlich isothermal equation to make fitting of the adsorption isothermal data of fresh calcium phosphate for gallic acid in different concentration's sucrose solution. The results are shown in figure 6 and the fitting parameters of different models are shown in table 2 and table 3.

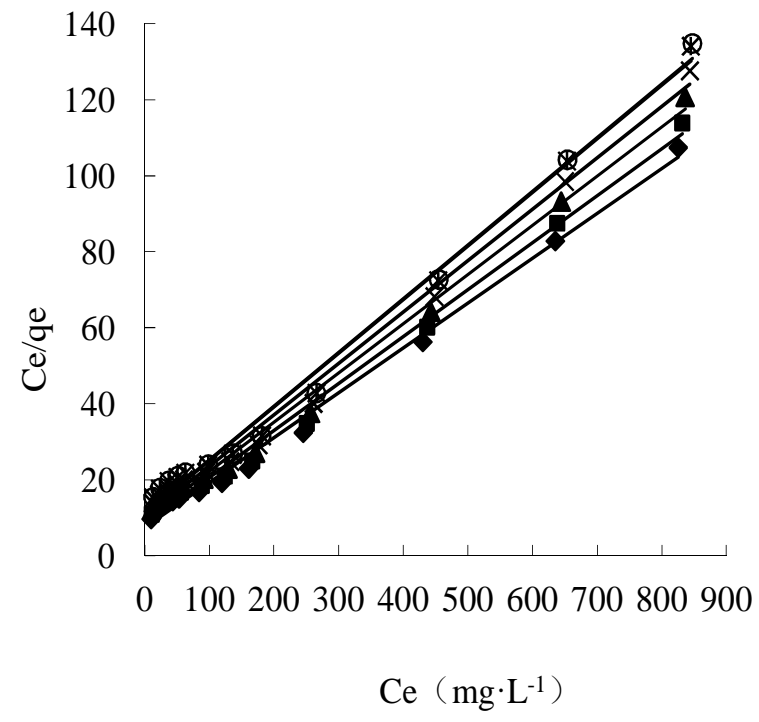

(a)

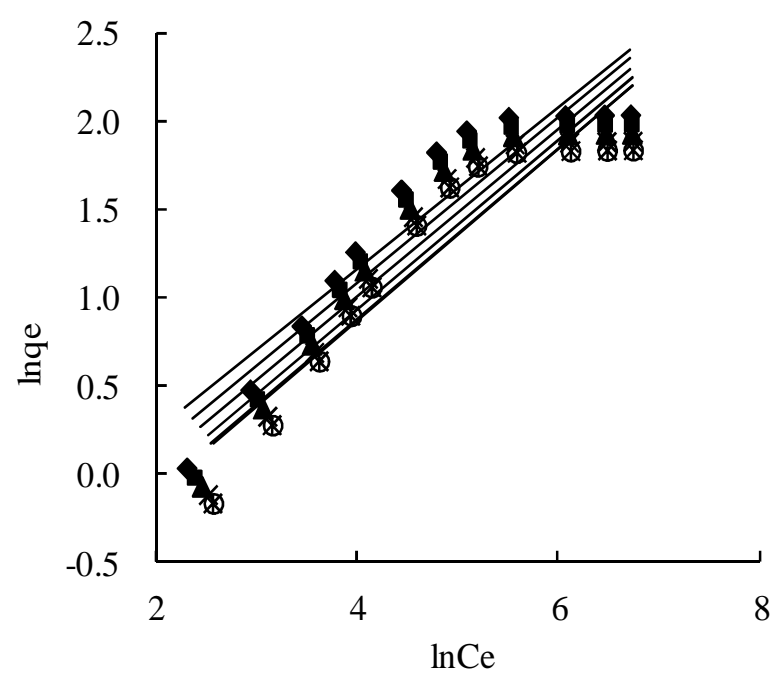

(b)

$-0.0 \%$

$-0.1 \%$

$-0.5 \% ; \times-1.0 \% ; *-5.0 \% ; 0-10.0 \%$

Fig.6 Linear fit of Langmuir isotherm equation(a)and Freundlich isotherm equation(b) in different concentrations of sucrose

Table.2 Langmuir isothermal parameters for adsorption of gallic acid on fresh calcium phosphate in different concentrations of sucrose

\begin{tabular}{ccccc}
\hline Sucrose concentration & equation & $\begin{array}{c}\mathrm{q}_{\mathrm{m}} \\
\left(\mathrm{mg} \cdot \mathrm{g}^{-1}\right)\end{array}$ & $\mathrm{k}_{\mathrm{L}}$ & $\mathrm{R}^{2}$ \\
\hline $0.0 \%$ & $\mathrm{Ce} / \mathrm{qe}=0.1186 \mathrm{Ce}+7.5694$ & 8.4317 & 0.0157 & 0.9965 \\
$0.1 \%$ & $\mathrm{Ce} / \mathrm{qe}=0.1236 \mathrm{Ce}+8.2172$ & 8.0906 & 0.0150 & 0.9939 \\
$0.5 \%$ & $\mathrm{Ce} / \mathrm{qe}=0.1296 \mathrm{Ce}+9.1785$ & 7.7160 & 0.0141 & 0.9932 \\
$1.0 \%$ & $\mathrm{Ce} / \mathrm{qe}=0.1352 \mathrm{Ce}+10.036$ & 7.3964 & 0.0135 & 0.9927 \\
$5.0 \%$ & $\mathrm{Ce} / \mathrm{qe}=0.1411 \mathrm{Ce}+10.993$ & 7.0872 & 0.0128 & 0.9921 \\
$10.0 \%$ & $\mathrm{Ce} / \mathrm{qe}=0.1413 \mathrm{Ce}+11.102$ & 7.0771 & 0.0127 & 0.9919 \\
\hline
\end{tabular}

Table. 3 Freudlich isothermal parameters for adsorption of gallic acid on fresh calcium phosphate in different concentrations of sucrose

\begin{tabular}{ccccc}
\hline Sucrose concentration & equation & $1 / \mathrm{n}$ & $\mathrm{k}_{\mathrm{F}}$ & $\mathrm{R}^{2}$ \\
\hline $0.0 \%$ & lnqe $=0.4513 \ln C \mathrm{e}-0.6760$ & 0.4513 & 0.5086 & 0.8924 \\
$0.1 \%$ & lnqe $=0.4687 \ln C \mathrm{e}-0.7986$ & 0.4687 & 0.4500 & 0.8763 \\
$0.5 \%$ & lnqe $=0.4759 \ln \mathrm{Ce}-0.9023$ & 0.4759 & 0.4056 & 0.8764 \\
$1.0 \%$ & lnqe $=0.4810 \ln \mathrm{ln}-0.9863$ & 0.4810 & 0.3730 & 0.8763 \\
$5.0 \%$ & lnqe $=0.4865 \ln \mathrm{ln}-1.0728$ & 0.4865 & 0.3420 & 0.8777 \\
$10.0 \%$ & lnqe $=0.4875 \ln \mathrm{ln}-1.0824$ & 0.4875 & 0.3388 & 0.8767 \\
\hline
\end{tabular}

Zhang Yan etc. had taken research on the adsorption and decolourization rules of calcium phosphate precipitation on sucrose solution and they found that the adsorption property of fresh calcium phosphate on caramel, melanoid and alkaline glucose degradation product in aqueous phase 
and sucrose solution all belong to Langmuir type of adsorption, and its linear correlation coefficient of fitting equation are greater than 0.9800 [12]. The results of researches made by Xu Yong etc. about the decoloration rules of new calcium phosphate on the pigments contained in enzymolysis Xylo-oligosaccharide liquid show that, the adsorption of fresh calcium phosphate on pigments also belongs to the Langmuir isothermal adsorption[13], which is consistent with the research conclusion in this paper.

From the fitting result, we can know that with the increase of sucrose concentration, $k$ reduced, which shows clearly that the existence of sugar molecules reduces the adsorption performance of fresh calcium phosphate for gallic acid and sucrose molecules has the inhibiting effect on the adsorption of fresh calcium phosphate on gallic acid. The causes of this phenomenon is related to that fresh calcium phosphate has selective adsorption capacity and the inherent characteristics of gallic acid. Because in the sucrose solution system, fresh calcium phosphate will produce adsorption for gallic acid and sucrose molecules. Therefore, sugar molecules and gallic acid will compete for the adsorption surface of fresh calcium phosphate. So, in sucrose solution system, the adsorption quantity of fresh calcium phosphate for gallic acid is smaller than the system without sucrose. However, for the system containing sugar and gallic acid, as a result of the different inherent characteristic of gallic acid and sugar molecules and different selective adsorption ability of different and fresh calcium phosphates, when the sucrose content is lower, adsorption quantity of sugar molecules on the surface of fresh calcium phosphate may not be able to be saturated, so the number of fresh calcium phosphate adsorbing sucrose molecules increases with the increase of sucrose content increase, thus reduce the adsorption quantity for gallic acid. But when the sucrose content exceeds critical region, the adsorption of fresh calcium phosphate for sugar molecules may have reached saturation, occupying the same proportion of adsorption surface. At this time, the increase of sucrose concentration will not make it adsorb more sugar molecules, as a result, make the adsorption quantity of fresh calcium phosphate for gallic acid reaches unanimity.

\section{Conclusion}

The adsorption kinetics of fresh calcium phosphate for gallic acid conforms to pseudo-second order kinetic equation, the range of fitting correlation coefficient $\mathrm{R}^{2}$ by using the pseudo-second order kinetic model is $0.9908 \sim 0.9964$. When the initial concentration of gallic acid are 25, 50 and 75 $\mathrm{mg} \cdot \mathrm{L}^{-1}$, adsorption rate constant of fresh calcium phosphate for gallic acid are $0.0614,0.0368,0.0149$ $\mathrm{g} \cdot \mathrm{mg}^{-1} \cdot \mathrm{min}^{-1}$ and its theoretical adsorption quantity are respectively $0.7556,1.3024$ and $2.0288 \mathrm{mg} \cdot \mathrm{g}^{-1}$. The existence of sucrose molecules does not affect the adsorption dynamics model of fresh calcium phosphate, however, the adsorption rate of fresh calcium phosphate for gallic acid will decrease for the existence of sucrose and the time of reaching to adsorption equilibrium has certain extension.

The adsorption of fresh calcium phosphate for gallic acid shows the monolayer adsorption and it is aligned with Langmuir isotherm adsorption model. Sucrose concentration has certain influence on the adsorption quantity of fresh calcium phosphate for gallic acid, besides, the adsorption quantity of that in the system containing no sugar is the largest. With the increase of sucrose solution concentration, the adsorption quantity of fresh calcium phosphate for gallic acid gradually reduces. When the concentration of the sucrose solution are $0 \%, 0.1 \%, 0.5 \%, 1.0 \%, 5.0 \%$ and $10.0 \%$ respectively, the saturated adsorption quantity of fresh calcium phosphate for gallic acid ordinally reduce to 8.4317 , 8.0906, 7.7160, 7.3964, 7.0872 and $7.0771 \mathrm{mg} \cdot \mathrm{g}^{-1}$.

\section{Acknowledgements}

The work was supported by Research Program of Science at Universities of Guangxi Autonomous Region (ZD2014077), Guangxi Science Foundation (2016GXNSFBA380073). 


\section{References}

[1] Q. Xu, G.J. Lu, G.D. Bian, G.D. Jin, W. Wang, X.Y. Hu, Y. Wang and Z.J. Yang: Materials Science and Engineering C, Vol. 32 (2012), p. 470

[2] H. El Boujaady, A. El Rhilassi, M. Bennani-Ziatni, R. El Hamri, A. Taitai and J.L. Lacout: Desalination, Vol. 275 (2011), p. 10

[3] X.P. Luo, L.X. Li, C.L. He and X.M. Zhao: Journal of Sichuan Agricultural University, Vol. 34(1) (2016), p. 60

[4] C. Y. Li, D.L. Li, Y. Zhang, S. Jiang, Z.Y. Du and K. Zhang: Food Fermentation Industries, Vol. 334 (2015), p. 125

[5] X. Xu, B.Y. Gao, W.Y. Wang, Q.Y. Yue, Y. Wang and S.Q. Ni: Colloids and Surfaces B:Biointerfaces, Vol. 70(2009), p. 46

[6] M.I. Konggidinata, B. Chao, Q. Lian, R. Subramaniam, M. Zappia and D.D. Gang: Journal of Hazardous Materials, Vol. 336 (2017), p. 249

[7] C.H. Ooi, W.K. Cheah, Y.L. Sim, S.Y. Pung and F.Y. Yeoh: Journal of Environmental Management, Vol. 197 (2017), p. 199

[8] A. Regti, H.B.E. Ayouchia, M. R. Laamari, S. E. Stiriba, H. Anane, M. E. Haddad. Applied Surface Science, Vol. 390 (2016) p. 311

[9] M. Triki, H. Tanazefti and H. Kochkar: Journal of Colloid and Interface Science, Vol. 493 (2017), p. 77

[10] M.A. Badawi, N.A. Negm, M.T.H. Abou Kana, H.H. Hefni and M.M. Abdel Moneem: International Journal of Biological Macromolecules, Vol. 99 (2017), p. 465

[11] Y.H. Tian, X.L. Xu, L.J. Li, and Q. Zhou: Science and Technology of Food Industry, Vol. 35(8)(2014), p. 126

[12] Y. Zhang, L. Li, M. Y. Cai and S.Y. Guo: Journal of Zhengzhou Institute of Technology, Vol. 22(1)(2001), p.73

[13] Y. Xu, S.Y. Yu, Q. Yong and C.C. Yao: Food Science, Vol.24(3)(2003), p. 19 
